\title{
Propagation of phytosanitary clones by in vitro culture
}

\author{
Marcela Dubchak, Olga Sultanova, and Viktor Bondarchuk \\ Scientific and Practical Institute of Horticulture, Viticulture and Food Technologies, Kishinev, \\ Moldova
}

\begin{abstract}
This article presents the process of accelerated reproduction of healthy clones of grapes, including the following stages: growing young shoots of the original plants of clones, introducing tops into in vitro culture, microclonal cuttings, adaptation of microplants to ex vitro culture, transplanting into cassettes with a soil substrate, transferring plants to a greenhouse for growing to the condition of vegetative seedlings and planting in a pre-propagation mother stock. For the successful implementation of each of the above operations in the SPIHVFT, a Cultural Complex has been equipped, consisting of a number of interconnected premises: a sterile box, a culture chamber and a vegetation chamber. The use of this Complex allows multiplying the required number of plants during the year, to grow vegetative seedlings by the spring of the next year and plant them in the prepropagation "Pre-base" mother plant. After a year, grafted vegetative seedlings grown from the vines of the mother plant were used for laying the mother stock.
\end{abstract}

\section{Introduction}

The period from the breeding of a new clone (variety) to the mass laying of production plantations of grapes depends on the rate of its reproduction and the creation of mother plantations. First, this applies to autochthonous and new varieties of grapes. In viticulture, there is a wide range of methods for propagating the vine: the method of dry table grafting, green and dry grafting directly on the plantation, dry and green cuttings. Each method has its own advantages and disadvantages. However, the method of microclonal grafting in vitro has become the most widespread in terms of accelerated reproduction of both scion and rootstock grape varieties [1-5]. The advantage of the method is a high reproduction rate, and the ability to grow plants under controlled conditions for a long period of time [5-10]. This method allows obtaining for a short period of time the required number of plants both for creating a collection of varieties (clones) and for planting mother stock plantations [11,12].

\section{Materials and methods}

The material for propagation was the vine of the original grape plants, free from viral, phytoplasmic diseases and bacterial cancer [13] (phytosanitary clones) of the following varieties: Moldova, Florichica, Feteasca nyagra, Plavai, Moldavian Kishmish, Radiant 
Kishmish, Kopchak, Krymoshie, Apiren roses, Legend and rootstock varieties PxR101-14, BxR SO4 and BxR Kober 5 BB.

Diagnosis of viral infection in grapevine was carried out by the ELISA-test method according to Clarc and Adams.

To diagnose the latent form of bacterial cancer, a microbiological test was used, consisting in inoculation of samples on a selective medium Roy and Sasser, followed by identification of the pathogen.

For prophylactic purposes, the vine, selected according to the results of tests of grape protoclones, was processed by the method of hot water therapy, in a laboratory thermostat LP-516, type 1387, at a temperature of $51^{\circ} \mathrm{C}$ for 45 minutes.

The explants were sterilized with $10 \%$ calcium hypochlorite solution for $5 \mathrm{~min}$ before being introduced into the in vitro culture. followed by 3 times 10 minutes washing with boiled distilled water. Initially, explants were planted on agar nutrient medium of the following composition: modified Murashige and Skoog medium with $1 / 2$ concentration of inorganic salts, IAA-1 mg/1, 2Ip-05 mg/l at PH-6.2, and agar-4.5 g/1. Further microclonal propagation of microplants was carried out on an ion exchange substrate Bion 311 (made in Belarus) in plastic containers. Microplants were grown in a culture room at a temperature of $26 \pm 1{ }^{\circ} \mathrm{C}$, an air humidity of $85 \%$ and a duration of illumination of 16 hours a day.

\section{Discussion and results}

\subsection{Growing young shoots of the basic clones}

Material for introduction into in vitro culture - the tops of green shoots were taken during the period of their active growth. For this, the vine of healthy protokles (bushes), which remained after testing for virus carriers and bacterial cancer, was cut into 2-ocellular cuttings in the second half of January and, after heat treatment, planted in pots with a soil substrate. To accelerate the processes of plant growth and development, the pots with cuttings were placed in climatic chambers with the KK 500 FIT phytotron system with the following parameters: 16-hour illumination, type 840 illumination color to simulate daylight: $280 \mu \mathrm{mol} / \mathrm{m}^{2} / \mathrm{sec}$ and temperature inside the chamber $25^{\circ} \mathrm{C}$. On the seventh day after planting in the pots, the buds of the cuttings swelled, and on the 14th day they bloomed (fig. 1). After 30 days, in mid-February, when the plants entered the active phase of growth, the first selection of tops was carried out for introduction into in vitro culture. Simultaneously with the removal of the tops, the green shoots were pruned to the 4-5th true leaf, in order to obtain actively growing stepchildren and thus increase the number of tops by 4-5 times for subsequent introduction into in vitro culture.

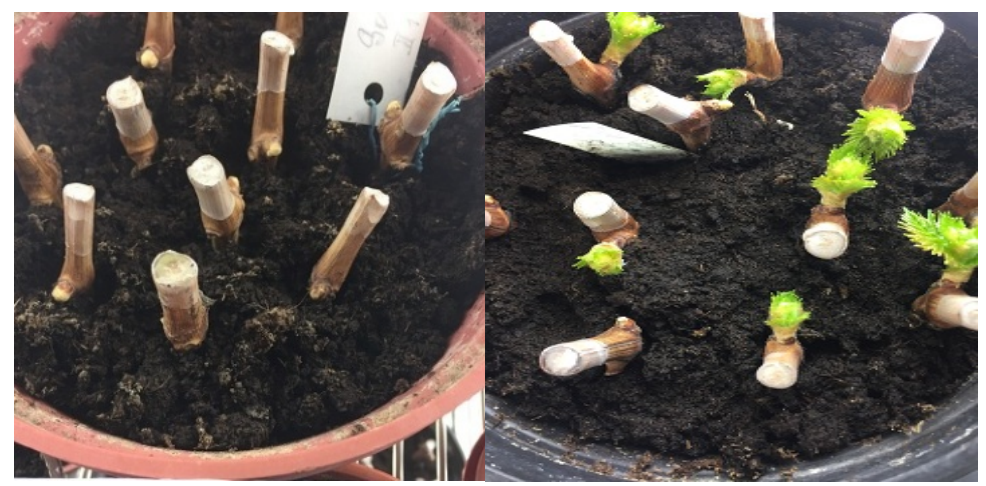

Fig. 1. Vine cuttings underwent hot water therapy in potting soil. Left - swelling of the buds 7 days after treatment; on the right - budding on the 14th day after hot water therapy 


\subsection{Introduction into in vitro culture}

The introduction into in vitro culture was carried out in a sterile box of the Culture complex equipped with bactericidal lamps and a laminar. The tips were planted on an agar medium. One explant was planted in glass test tubes, and three in plastic containers. On each test tube and container, an inscription was made indicating the variety and the date of planting, after which they were transferred to an adjacent room - a culture chamber.

\subsection{Growing plants in in vitro culture}

The cultivation of plants in in vitro culture was carried out in a specially equipped culture chamber, in which a constant temperature and humidity were automatically maintained. Containers and test tubes with plants were placed on racks mounted in 3 tiers. The area of the shelves allows placing 1500 containers - about $4500-5000$ pieces of plants at a time (fig. 2 ). Additional illumination is provided by ice lamps of the blue $(440-460 \mathrm{~nm})$ spectrum and red (630-660nm) spectrum with an intensity of 5000-6000 lux [14,15]. This type of lighting promotes the rapid emergence of the first roots and the good development of the explant root system. Cultivation of microplants under blue and red lamps had a beneficial effect on their growth and development, which significantly increased the efficiency of propagation by microclonal cuttings in vitro [8]. Therefore, in the conditions of the culture chamber, in such varieties as Moldova, Florichica, Feteasca Nyagra, Plavai, Kopchak, root formation began on the 7-9th day, and after 30-35 days, the test tube plants reached the necessary development for microcutting. Seedless varieties: Moldavian Kishmish, Radiant Kishmish, Apiren roses formed roots on the 12-15th day and reached the size of 6-8 internodes on the 45-50th day. Thus, the variety specificity to the medium of Murashige and Skoog was revealed, on which the period of plant development varied from 30 to 50 days [13].

Microclonal cuttings were carried out only in plastic containers with ion exchange substrate Bion 311 (fig. 3). Microplants on an ion exchange substrate developed much faster (within 30-35 days) than on an agar medium (35-50 days) and formed a good root system. At the beginning of May, the first plants obtained by the method of micropropagation were exposed for adaptation (fig. 3).

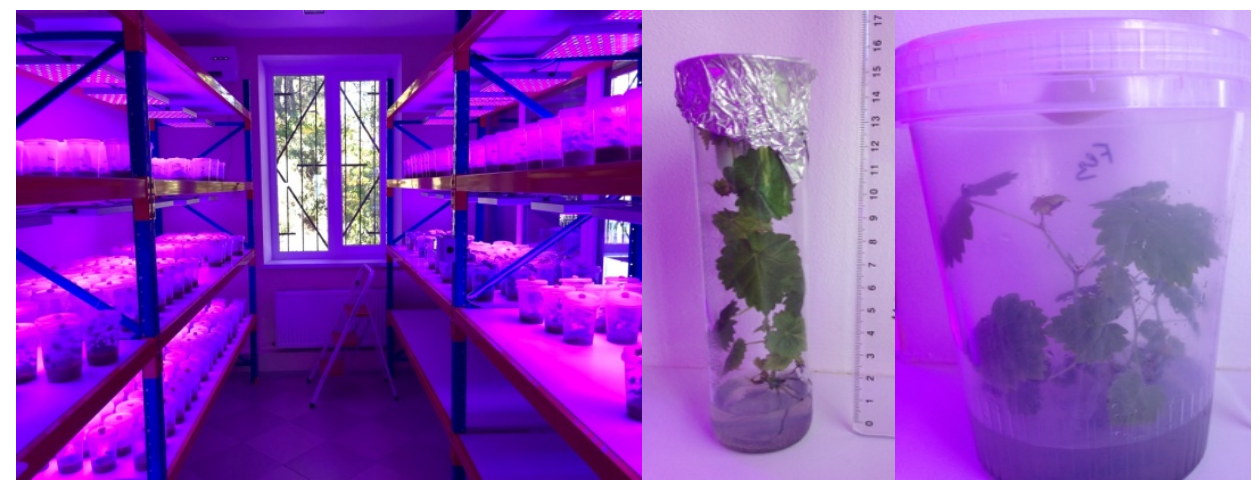

Fig 2. The development of microplants of grapes in in vitro culture in the culture room - on the left; on agar culture medium in glass test tubes - in the center; on the right - in plastic containers.

It should be noted that both in test tubes and in containers, the growth and development of plants proceeded in the same way. Within 35-50 days after being introduced into the in vitro culture, depending on the cultivar, the explants grew into microplants with 6-8 nodes and a developed root system (Fig. 2). The disadvantage of container cultivation is that in case of infection of one of the three plants, it is necessary to remove the affected plant under sterile 
conditions, if possible, or to transplant the non-infected into another container. When an infection appeared in a test tube, the plant was removed along with the medium. Lack of test tubes - high cost, the need for careful handling and the possibility of cracking.

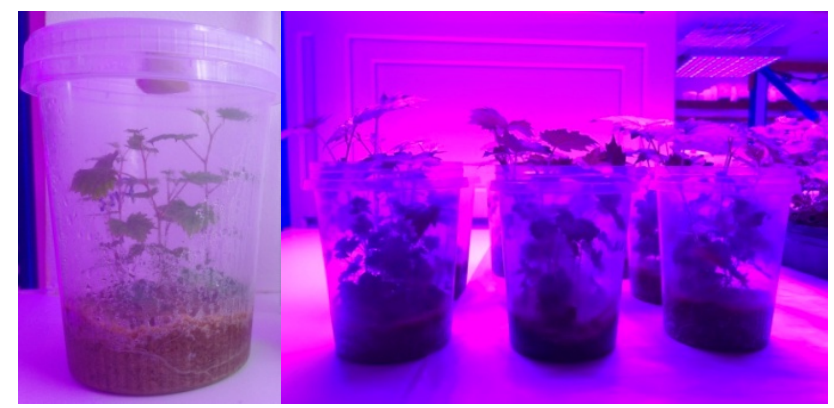

Fig. 3. Microclonal grape cuttings in containers: on the left - the development of microplants on Bion 311 ; on the right - plant adaptation to ex vitro conditions

\subsection{Transfer to ex vitro conditions}

For the successful transfer of microplants to ex vitro conditions, they were adapted to environmental conditions. For this, the containers in which the plants reached 6-8 internodes were opened, and they further developed in the conditions of the culture chamber for 7-14 days. The Bion 311 substrate, in contrast to the agar medium, was not infected after opening the container, which greatly simplifies the adaptation process. During the adaptation period, the plants grew outside the container, and the leaves acquired the pubescence characteristic of the variety.

The containers with the plants that had undergone the adaptation process were transferred to the growing chamber (fig.4). The climatic conditions in the growing chamber are similar to those of the culture chamber, so the plants do not experience stress from being transferred to another room. In the growing chamber, the plants from the containers were planted into cassettes with a soil substrate. Favorable conditions of the culture chamber promoted rapid engraftment in the soil substrate, as a result of which, after a month, they were transferred without damage to the greenhouse for further cultivation. If the transfer of plants to cassettes with a substrate was carried out in the spring-summer period, then 15-20 days after being in the culture chamber, the plants in the cassettes were transferred to the greenhouse for further cultivation. In the case when the transfer to cassettes with a soil substrate was carried out in the autumn-winter period, the plants were left in the culture chamber until spring.

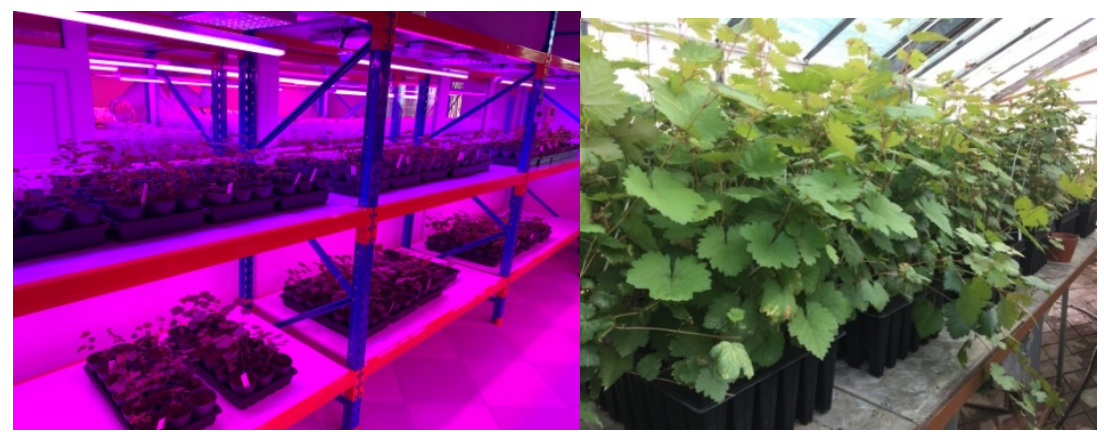

Fig. 4. Growing plants: on the left - in the growing chamber in cassettes with soil substrate; on the right - in a greenhouse 


\subsection{Growing plants in a greenhouse}

The greenhouse, into which the grape plants were transferred from the culture chamber, is equipped for year-round keeping of plants and growing vegetative seedlings suitable for planting in the mother stock. The greenhouse is heated during the winter. The minimum temperature in winter is $+5^{\circ} \mathrm{C}$ at night, during the day $+10-15^{\circ} \mathrm{C}$ and above, which favorably affects the ripening of the vine and the passage of natural leaf fall. In February, grape plants were pruned by 2-3 eyes to obtain one well-developed shoot.

Favorable conditions for the development of plants in the greenhouse in the springsummer period are carried out by covering with a sun-protection net, forced exhaust ventilation systems, fine water spray, operating in automatic mode. Treatments are systematically carried out to prevent the appearance of fungal diseases, foliar and root feeding. The use of this technology during 2017-2018 made it possible to obtain 3132 pieces of vegetative seedlings of scion varieties: Moldavian Kishmish, Apiren roses, Krymposhie, Plavai, Legenda, Copchak, Moldova; in 2019-2020 - 2408 pieces of vegetative seedlings of varieties: Feteaske regale, Feteaske alba, Sauvignon, Merlot, Black Kaushansky, Telti Kuruk, Tudor, Radiant Kishmish and 1210 pieces of rootstock varieties: BxR Kober 5BB, BxR CO4 and PxR 101-14, and in 2020-2021 - 733 pieces of scion and 905 pieces of rootstock varieties. Vegetable seedlings grown in a greenhouse were planted in a pre-propagation mother stock "Pre-base».

\subsection{Mother stocks of grape vine}

The mother stock "Pre-base" is laid by self-rooted vegetative seedlings grown from grape plants as a result of microclonal propagation. The purpose of the mother stock is to grow a vine suitable for obtaining grafted grape seedlings. With good agrotechnical care, highquality vines suitable for grafting can be obtained within a year after planting. Therefore, from those planted in 2018 in the amount of 3132 pieces vegetative seedlings, in the fall of 2020 a vine was harvested, and in the spring of 2021, grafted vegetative seedlings were grafted and grown in the amount of 11,250 pieces

In June 2021, with these seedlings, a mother stock of the biological category "Basic" was planted on an area of 4.9 hectares. The purpose of the mother stock is to grow a high-quality scion vine, free from viral, phytoplasmic diseases, and bacterial cancer, for the production of grafted grape seedlings of the biological category "Certified" and planting industrial grape plantations by them.

\section{Conclusion}

The use of microclonal cuttings in in vitro culture for the propagation of phytosanitary clones of grapes allows planting a mother stock with grafted seedlings for the 5th year from the start of reproduction. The high efficiency of reproduction in in vitro culture is ensured by favorable conditions of the Cultural Complex, the use of blue-red lighting and Biony 311, which contribute to the development of a powerful root system, strong growth, full adaptation to the external environment, and, as a result, $95-100 \%$ survival rate of plants when planted in soil.

\section{References}

1. E.A. Baimukhametova, B.R. Kuluev, Biotekhn., 36(2), 26-42 (2020) http://doi.org/10.21519/0234-2758-2020-36-2-26-42 
2. F. K. Pilatti, T. Aguiar, T. Simões, E. E. Benson, A. M. Viana, In Vitro Cell.Dev.Biol.Plant, 47, 82-98 (2011) https://doi.org/10.1007/s11627-010-9302-y

3. K.A. Quiroz, M. Berríos, B. Carrasco, J.B. Retamales, P.D.S. Caligari, R. GarcíaGonzáles, Biol. Res., 50, 20 (2017) https://doi.org/10.1186/s40659-017-0125-8

4. S. Kazybayeva, I. Kovalchuk, T. Turdiyev, S. Kulshanov, L. Azhitayeva, BIO Web Conf., 25, 05003 (2020) https://doi.org/10.1051/bioconf/20202505003

5. O. Seget, I. Avdeenko, G. Aleynikova, G. Malih, BIO Web Conf., 25, 05010 (2020) https://doi.org/10.1051/bioconf/20202505010

6. A. Batukaev, M. Batukaev, D. Palaeva, E. Sobralieva, S. Sushkova,. International Multidisciplinary Scientific GeoConference, 19, 727-736 (2019) http://doi.org/10.5593/sgem2019/6.1/S25.094

7. E. A. Sobralieva, M. S. Batukaev, D. O. Palaeva, Theor. Appl. Probl. AI, 1(39), 26-29 (2019) http://www.nitu.ru/tppapk/19 1.pdf

8. A. N. Rebrov, N. P. Doroshenko, L. P. Troshin, Науч. Жур. КубГАУ, №124(10), (2016) http://ej.kubagro.ru/2016/10/pdf/08.pdf

9. C.M. Ruedell, M.R. de Almeida, J. Schwambach, C.F. Posenato, A.G. Fett-Neto, Plant Growth Regul., 69, 235-245 (2013) https://doi.org/10.1007/s10725-012-9766-3

10. L. V. Tashmatova, O.V. Matsneva, T. M. Khromova, V. V. Shakhov, E3S Web Conf., 254, 04001 (2021) https://doi.org/10.1051/e3sconf/202125404001

11. A. Rebrov, BIO Web Conf., 25, 05005 https://doi.org/10.1051/bioconf/20202505005

12. L. Samarina, T. Kolomiyets, V. Malyarovskaya, S. Gubaz, N. Platonova, Plant Tiss. Cult. Biotechnol., 26, 97-104 (2016) https://doi.org/10.3329/ptcb.v26i1.29771

13. L.G. Bratkova, N.N. Cacenko, Achiev. Sci. Tech. AIC, 29(6), 49-52 (2015) http://agroapk.ru/59-archive-en/06-2015-en/906-2015-06-13-en

14. F. Kumsa, Int. J. Agril. Res. Innov. Tech., 10(1), 1-5 (2020) https://doi.org/10.3329/ijarit.v10i1.48087

15. F. Fallah, D. Kafrizi, J. of App. Biot. Rep., 3(4), 495-499 (2016) http://www.biotechrep.ir/article_69241.html 